\title{
Comparison of DNA methylation profiles in human fetal and adult red blood cell progenitors
}

\author{
Samuel Lessard ${ }^{1,2}$, Mélissa Beaudoin ${ }^{1}$, Karim Benkirane ${ }^{3}$ and Guillaume Lettre ${ }^{1,2^{*}}$
}

\begin{abstract}
Background: DNA methylation is an epigenetic modification that plays an important role during mammalian development. Around birth in humans, the main site of red blood cell production moves from the fetal liver to the bone marrow. DNA methylation changes at the $\beta$-globin locus and a switch from fetal to adult hemoglobin production characterize this transition. Understanding this globin switch may improve the treatment of patients with sickle cell disease and $\beta$-thalassemia, two of the most common Mendelian diseases in the world. The goal of our study was to describe and compare the genome-wide patterns of DNA methylation in fetal and adult human erythroblasts.
\end{abstract}

Methods: We used the Illumina HumanMethylation 450 k BeadChip to measure DNA methylation at 402,819 CpGs in ex vivo-differentiated erythroblasts from 12 fetal liver and 12 bone marrow CD34+ donors.

Results: We identified 5,937 differentially methylated CpGs that overlap with erythroid enhancers and binding sites for erythropoiesis-related transcription factors. Combining this information with genome-wide association study results, we show that erythroid enhancers define particularly promising genomic regions to identify new genetic variants associated with fetal hemoglobin (HbF) levels in humans. Many differentially methylated CpGs are located near genes with unanticipated roles in red blood cell differentiation and proliferation. For some of these new candidate genes, we confirm the correlation between DNA methylation and gene expression levels in red blood cell progenitors. We also provide evidence that DNA methylation and genetic variation at the $\beta$-globin locus independently control globin gene expression in adult erythroblasts.

Conclusions: Our DNA methylome maps confirm the widespread dynamic changes in DNA methylation that occur during human erythropoiesis. These changes tend to happen near erythroid enhancers, further highlighting their importance in erythroid regulation and HbF production. Finally, DNA methylation may act independently of the transcription factor BCL11A to repress fetal hemoglobin production. This provides cues on strategies to more efficiently re-activate $\mathrm{HbF}$ production in sickle cell disease and $\beta$-thalassemia patients.

\section{Background}

DNA methylation is a dynamic epigenetic mark mostly found on cytosine residues of certain CPG dinucleotides in mammalian genomes. In humans, it is involved in gene imprinting, X-chromosome inactivation, and transposable element suppression [1]. DNA methylation is generally associated with transcriptional silencing and plays an essential role in maintaining stem cell pluripotency and

\footnotetext{
* Correspondence: guillaume.lettre@umontreal.ca

'Montreal Heart Institute, 5000 Bélanger Street, Montréal, Québec H1T 1C8,

Canada

${ }^{2}$ Faculté de Médecine, Université de Montréal, 2900 Boul. Édouard-Montpetit, Montréal, Québec H3T 1J4, Canada

Full list of author information is available at the end of the article
}

controlling other cell- or organ-specific developmental programs [1]. Despite the well-established role of DNA methylation during development, we are only now starting to collect single-base resolution maps of these developmentally regulated DNA methylation changes thanks to improvements in DNA array- and sequencing-based technologies [2]. Such precise DNA methylome maps are important in order to understand how transcriptional networks are controlled during development or how dys-regulation of DNA methylation - observed in human diseases like cancer or upon treatment with DNA demethylating agents - may impact cell functions and/or identity [3].

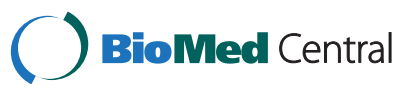

(C) 2015 Lessard et al.; licensee BioMed Central. This is an Open Access article distributed under the terms of the Creative Commons Attribution License (http://creativecommons.org/licenses/by/4.0), which permits unrestricted use, distribution, and reproduction in any medium, provided the original work is properly credited. The Creative Commons Public Domain Dedication waiver (http://creativecommons.org/publicdomain/zero/1.0/) applies to the data made available in this article, unless otherwise stated. 
Characterization of mouse models with conditional deletions of genes that encode DNA methyltransferases enzymes that catalyze the transfer of methyl groups onto DNA - has firmly established that DNA methylation is essential during hematopoietic stem cell differentiation and repopulation $[4,5]$. Recent reports used enrichment of methylated DNA followed by next-generation DNA sequencing to characterize genome-wide DNA methylation changes in murine hematopoietic stem and progenitor cells, and during erythropoiesis [6,7]. The studies showed a progressive DNA demethylation during erythroid differentiation, and confirmed that groups of CpGs in promoters - so-called CpG islands tend to be hypomethylated and correlated with active gene expression $[6,7]$.

Although large-scale projects are starting to generate comprehensive DNA methylation datasets [8], they do not capture all stages of human erythroid differentiation and proliferation. In an initial study, investigators monitored genome-wide changes in DNA methylation during human erythropoiesis of bone marrow-derived CD34+ progenitor cells and reported global DNA hypomethylation [9]. We are particularly interested in the epigenetic differences between fetal- and adult-stage erythroblasts that originate, respectively, from the fetal liver and the bone marrow. The large epigenomic projects (e.g. ENCODE, Roadmap Epigenomic, Blueprint) do not profile DNA methylation in these erythroid cells. During this erythroid transition, which occurs around birth in humans, erythroblasts reduce the production of fetal hemoglobin $(\mathrm{HbF})$ and increase the production of adult hemoglobin (HbA) through the transcriptionally regulated fetal-to-adult hemoglobin switch [10]. This gene expression switch is accompanied by progressive DNA hypermethylation of the HBG2 promoter, which encodes the $\gamma$-globin subunit of $\mathrm{HbF}[11,12]$. Understanding the molecular mechanism behind the fetal-to-adult hemoglobin switch is particularly important since re-activating $\mathrm{HbF}$ production is the most promising therapy for patients with sickle cell disease and $\beta$-thalassemia [10]. Ex vivo differentiation protocols now exist to cultivate sufficient number of fetal and adult human erythroblasts to extend the characterization of DNA methylation to the rest of the genome [13].

Here we provide comprehensive DNA methylome maps of human erythroblasts differentiated ex vivo from CD34+ progenitor cells purified from the fetal liver or the bone marrow. By analyzing DNA methylation values at 402,819 tested CpGs in fetal and adult erythroblasts, we identified 5,937 differentially methylated CpGs. These differentially methylated regions include the known $\beta$-globin locus, other genes with known roles in erythropoiesis, as well as several other genes with no previously recognized functions in red blood cell differentiation. We showed that the differentially methylated
CpGs cluster within stage-specific erythroid enhancers and are located near binding motifs for transcription factors that regulate hematopoiesis. Finally, we determined that DNA methylation and genetic variation at the $\beta$-globin locus independently control $\mathrm{HbF}$ production in adultstage cells.

\section{Methods}

\section{Cell culture and differentiation}

Primary human fetal and adult CD34+ hematopoietic stem/progenitor cells harvested from 24 anonymous donors (12 of each) were purchased from DV Biologics [14] and Lonza [15], respectively. Primary fetal and adult erythroblasts were generated using two-phase serumfree culture as described previously [13]. Briefly, primary human fetal and adult CD34+ cells are cultured in the expansion medium until day 6 . They are then transferred to the differentiation medium until cells are collected at day 18. The medium is changed every 3 days. We assessed cell morphology by Wright-Giemsa coloration, and measure cell size and count using the MOXI Z Mini automated cell counter (ORFLO technologies, ID), and hemoglobin production by capillary electrophoresis.

\section{Genomic DNA extraction and methylation assay}

We extracted genomic DNA with the Gentra Puregene Cell Kit (Qiagen). DNA was further precipitated with alcohol in order to obtain highly purified DNA. DNA bisulfite conversion was performed using the EZ DNA Methylation Gold Kit (Zymo Research, CA, USA). We used the Infinium HumanMethylation450 BeadChip (Illumina Inc., CA, USA) to measure genome-wide patterns of DNA methylation; the experiment was carried out at the Genome Quebec-McGill Innovation Centre using Illumina's recommended protocol. We assessed data quality with the minfi $R$ package [16] and normalized intensities using the ARRm software, which corrects for probe type, background, dye, and position effects [17]. We removed probes that target a genomic sequence annotated to carry genetic variants based on dbSNP version $137(\mathrm{~N}=82,694)$. DNA methylation data have been submitted and are available from the National Center for Biotechnology Information (NCBI) Gene Expression Omnibus (GEO) repository (accession number (GEO: GSE56491)). To identify single CpG that are differentially methylated between fetal and adult erythroblasts, we converted DNA methylation $\beta$-values into Mvalues [18] and used linear regression in $\mathrm{R}$, correcting for sex effects. To combine results across CpGs located within a functional genomic unit, we used a generalization of Fisher's method that takes into account correlation between nearby $\mathrm{CpGs}$ and that is already implemented in the RnBeads software [19]. We used RefSeq (release 61) coordinates to map $\mathrm{CpGs}$ to specific genes. Promoter 
CpGs are located within 1.5-kb upstream of a RefSeq gene and enhancer CpGs map to erythroid enhancers experimentally identified in ex vivo differentiated CD34+ cells [20]. In total, we could map 233,894 CpGs to 25,891 gene bodies, 130,854 CpGs to 25,103 promoters, and 11,709 CpGs to 4,604 erythroid enhancers. DNA methylation levels at the $H B G 2$ promoter were measured using the MassArray EpiTYPER platform (Sequenom Inc., CA, USA), which quantitatively analyze fragmented PCR products using MALDI-TOF mass spectrometry. Bisulfiteconverted DNA was amplified by PCR as previously described [21].

\section{RNA extraction and gene expression analysis}

RNA was extracted using the RNeasy Plus Mini Kit from Qiagen. We determined the quality and quantity of RNA using the RNA 6000 Nano kit on the Bioanalyzer instrument (Agilent). We converted RNA into cDNA with the High Capacity cDNA Reverse Transcription Kit (ABI) and performed quantitative PCR using the Platinum SYBR Green qPCR Mix (Invitrogen). Relative gene expression levels were measured using the $\Delta \Delta C_{t}$ method and we evaluated statistical significance with $t$-test as implemented in the $\mathrm{R}$ software (v. 3.0.0). Primer sequences are listed in Additional file 1: Table S1. Total RNA for RNA sequencing was extracted using the miRNEASY kit (Qiagen). Paired-end RNA sequencing was performed on the Illumina Hiseq 2000 platform. Reads were mapped to the genome using Tophat2 (v.2.0.9) and transcript abundances were estimated using Cufflinks (v.2.2.1).

\section{Enrichment analyses}

We used binomial tests to measure the enrichment of differentially methylated CpGs in gene bodies, promoters, and erythroid enhancers as compared to the rest of the genome surveyed by the Illumina HumanMethylation450 BeadChip. To compare the enrichment of differentially methylated $\mathrm{CpGs}$ in erythroid enhancers versus other enhancers present in different cell types, we obtained enhancer coordinates from nine cell lines analyzed by the ENCODE Project [22]. We used DAVID to measure the enrichment of gene ontology (GO) terms and biological pathways among genes with a mean $\beta$-value difference $>0.1$ and a combined $P<9 \times$ $10^{-7}$ in their body or promoter, taking into account the coverage of the Illumina HumanMethylation450 BeadChip [23,24]. To identify transcription factor binding motifs that are enriched at differentially methylated loci, we used the HOMER software and a list of 495 pre-defined transcription factor motifs [25], limiting the search to 200 base pairs on both sides of differentially methylated CpGs. For this analysis, we compared the 5,000 most hypomethylated regions in fetal erythroblasts to the
5,000 most hypomethylated regions in adult erythroblasts, as recommended. HOMER results were stable using different thresholds to select hypomethylated regions.

\section{DNA genotyping and association studies}

Genotyping was performed at the Pharmacogenomics Center of the Montreal Heart Institute using the HumanOmniExpress BeadChip array (Illumina). We performed quality-control steps with PLINK [26], removing markers with genotyping success $<90 \%$ or HardyWeinberg $P<1.0 \times 10^{-6}$. All samples had a genotyping success rate $>99.8 \%$. We imputed genetic variants with the $\mathrm{MaCH} /$ minimac software [27] and reference haplotypes from the 1000 Genomes Project (phase I). For association analysis, we only considered genotyped markers or markers with an imputation quality $\mathrm{r} 2$ hat $>0.6$. We tested association between SNP genotypes and methylation levels under an additive model using linear regression as implemented in R. For BCL11A, we tested CpGs inside the gene body or the promoter (chr2:60,678,30260,781,633). For $H B S 1 L-M Y B$, tested CpGs were either inside the gene bodies and promoters of the genes, or in the intergenic region (chr6:135,281,517-135,540,311). For the $\beta$-globin locus, all CpGs between the promoter of $H B E$ and the gene body of $H B B$ were included (chr11:5,246,696- 5,527,882).

To test the enrichment of $\mathrm{HbF}$ association signals with DNA methylation-implicated erythroid enhancers, we accessed genome-wide association study (GWAS) data of 1,140 African Americans from the Cooperative Study of Sickle Cell Disease (CSSCD). These individuals were genotyped on the Illumina Human610-Quad array, as previously described [28]. SNPs were phased using $\mathrm{MaCH}$ (v1.0.16) and imputed with haplotypes from European and African samples generated by the 1000 Genomes Project (phase I) using minimac (v4.4.3). Association $P$ values with $\mathrm{HbF}$ were calculated using mach2qtl (v1.0.8). In total, 6,994,357 SNPs were included in the analysis. We found 63,876 SNPs that overlap with 12,683 erythroid enhancers.

\section{Results and discussion}

\section{Ex vivo culture of erythroid progenitor cells}

We used a previously established two-phase cell culture protocol to expand and differentiate primary human fetal and adult CD34+ hematopoietic stem/progenitor cells into erythroblasts [13]. For each tissue (fetal liver or bone marrow), we differentiated CD34+ cells from 12 anonymous donors to compare not only DNA methylation changes between fetal and adult erythroblasts, but also between individuals within a developmental stage. We validated the expansion and differentiation cell culture protocol by characterizing cell size, growth, and morphology (Additional file 1: Figure S1). To determine 
if erythroblasts differentiated ex vivo maintain characteristics that are specific to their tissue of origin, we measured expression of genes involved in the fetal-to-adult hemoglobin switch and quantified hemoglobin production by capillary electrophoresis. As expected, erythroblasts derived from bone marrow express significantly more $B C L 11 A$ and $K L F 1$ than fetal erythroblasts (Additional file 1: Figure S2A-B). BCL11A and KLF1 are transcriptional repressors of $H B G 2$ gene expression; $K L F 1$ also increases $H B B$ gene expression, which encodes the $\beta$-globin subunit of $\mathrm{HbA}[13,29-31]$. Consistently, erythroblasts from the fetal stage produce exclusively $\mathrm{HbF}(100 \pm 0 \%)$ whereas differentiated cells from the adult stage produce mostly $\mathrm{HbA}(84.2 \pm$ 4.8\%) (Additional file 1: Figure S2C-E). Using a targeted assay that measures DNA methylation at seven CpGs within the HBG2 promoter, we also confirmed hyper- and hypomethylation of this promoter in adult and fetal erythroblasts, respectively (Additional file 1: Figure S3) [32]. Taken together, these results confirm that the erythroblasts have maintained their developmental stage specificity.

In the mouse and humans, it is known that global demethylation occurs during erythropoiesis $[6,7,9]$. For this reason, it is important to confirm that fetal and adult erythroblasts grow and differentiate under similar kinetics. To directly address this concern, we performed a time-course analysis of the expression of genes that code for markers of differentiation and that are localized at the cell surface of erythroblasts: CD34, CD71 (transferrin receptor (TRFC)), and CD235a (glycophorin A (GYPA)). Recent transcriptomic analyses in ex vivo-differentiated human erythroblasts have confirmed that the expression levels of these genes are strongly correlated with the localization of the encoded proteins at the cellular membrane $[33,34]$. As expected, CD34 is highly expressed at the beginning of differentiation and decreases thereafter, whereas the expression of $C D 71$ and $C D 235 a$ increases during differentiation (Additional file 1: Figure S4). At any given time point, the expression of these three genes was not different between fetal liver- and bone marrowderived CD34+ progenitors cells, except for CD235a at the beginning of differentiation (more expressed in fetal liver cells) (Additional file 1: Figure S4). These results confirm that fetal and adult erythroblasts grow and differentiate at a similar rate, and therefore that our experiment is adequate to detect differences in DNA methylation that are mostly due to the different developmental stage (fetal liver vs. bone marrow) of these cells.

\section{The DNA methylation landscape in human erythroblasts}

We used the Illumina HumanMethylation $450 \mathrm{k}$ BeadChip to measure quantitatively DNA methylation across the genome in ex vivo-differentiated erythroblasts from
12 fetal liver and 12 bone marrow donors. After quality control and intensity normalization steps, we obtained DNA methylation $\beta$-values (from 0.0 (unmethylated) to 1.0 (fully methylated)) for 402,819 CpGs (Additional file 1: Figure S5). We only used these CpGs in subsequent analyses. We used DNA methylation data generated by the Roadmap Epigenomics Project [35] using reduced representation bisulfite DNA sequencing (RRBS) from mobilized adult CD34+ cells to validate the DNA methylation levels measured in erythroblasts with the Illumina HumanMethylation $450 \mathrm{k}$ BeadChip. Although not differentiated ex vivo, we reasoned that these CD34+ cells would be a good proxy for adult erythroblasts used in our experiment. Across 80,136 CpGs available in both datasets, we observed a strong correlation of DNA methylation values (Pearson's $r=0.91, P<2.2 \times 10^{-16}$, Additional file 1: Figure S6A). Importantly, we repeated the same analysis with other RRBS datasets from the Roadmap Epigenomics Project. We observed that DNA methylation values in erythroblasts are more correlated with DNA methylation data from blood-related rather than blood-unrelated cells or tissues (Additional file 1: Figure S6B). These comparisons confirm the quality and specificity of the DNA methylation data generated with the Illumina $450 \mathrm{k}$ array in human erythroblasts.

We analyzed DNA methylation across all CpGs tested using unsupervised clustering methods. The two main clusters accurately distinguish fetal liver- from bone marrow-derived erythroblasts (Figure 1). This result confirms our hypothesis that changes in DNA methylation captures developmental differences between fetal and adult erythroblasts. We also noted that the two main stage-specific clusters are sub-divided according to the sex of the sample's donors (Figure 1). This sexspecific clustering is mostly dependent on DNA methylation values at $\mathrm{CpGs}$ located on the $\mathrm{X}$-chromosome.

\section{Differential DNA methylation between fetal and adult erythroblasts}

We sought to identify differentially methylated single CpG sites by comparing methylation $\beta$-values for fetal and adult erythroblasts, taking into account potential sex effect. We used two criteria to define differentially methylated CpGs: a difference in $\beta$-values of $\geq 0.2$ and a $P \leq 1.25 \times 10^{-7}$ (significant threshold after Bonferonni correction). Using this definition, we found 5,937 differentially methylated CpGs (Figure 2A and Additional file 2: Table S2).

We discuss below two interesting examples of differentially methylated loci detected in human erythroblasts. GCNT2 encodes the enzymes responsible for the conversion of the blood $\mathrm{i}$ - to the I-antigen during the fetalto-adult transition in erythroblasts. GCNT2 codes for three isoforms: isoform $\mathrm{C}$ is responsible for the I-antigen 

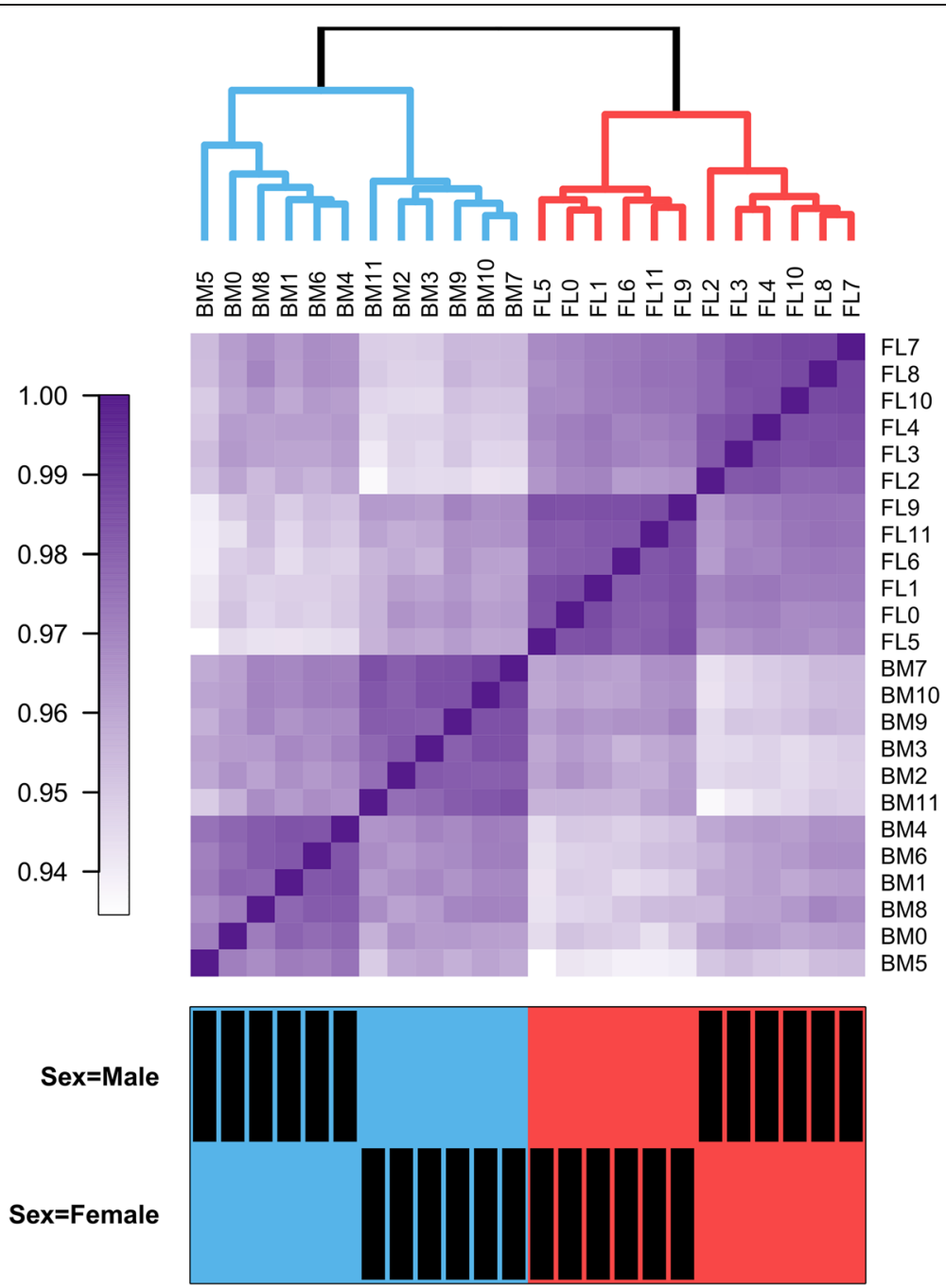

Figure 1 Hierarchical clustering analysis of DNA methylation in erythroblasts. Unsupervised clustering analysis of DNA methylation at 402,819 CpGs separates precisely fetal liver-derived from bone marrow-derived erythroblasts. Within each developmental stage, cells are sub-divided by the sex of the donors. The top panel represents the dendogram of the different clusters identified. The middle panel summarizes pairwise correlations between all samples. In the bottom panel, black rectangles identify male and female donors. BM: adult erythroblasts; FL: fetal erythroblasts.

phenotype in adult erythrocytes [36]. In our cell culture system, we found that cg14322298 in the promoter of isoform $C$ is hypomethylated in adult erythroblasts $\left(\Delta \beta_{\mathrm{a}}\right.$ dult-fetal $=-0.47, P=8.0 \times 10^{-16}$; Figure $\left.3 \mathrm{~A}\right)$. We found that GCNT2-C expression levels correlate with DNA methylation at cg14322298 in erythroblasts (Figure 3B). Isoform $\mathrm{B}$ had very low expression in both fetal and adult erythroblasts, whereas isoform $\mathrm{A}$ and $\mathrm{C}$ explained most of GCNT2 expression in fetal (72.2\%) and adult (75.1\%) erythroblasts, respectively (Additional file 1: Figure S7). Another example of differentially methylated loci include CpGs in the ARID3A/Bright gene that show a marked differential DNA methylation pattern, being almost completely methylated in fetal-stage cells (e.g. $\quad$ cg08894487, $\Delta \beta_{\text {adult-fetal }}=-0.93, \quad P=3.8 \times 10^{-21}$ ) (Figure $3 \mathrm{C}$ ). This gene is required for hematopoietic stem cell development: definitive erythrocyte formation in the fetal liver is impaired in Bright knockout mice, but not at the primitive (embryonic) stage [37]. ARID3A expression was higher in fetal erythroblasts (Figure 3D). Positive correlations between DNA methylation inside gene bodies and gene expression levels have been reported previously [38]. Overall, we observed a global enrichment of positive correlations between DNA methylation at $\mathrm{CpGs}$ inside gene bodies and expression levels in erythroblasts. In contrast, there is a marked enrichment of negative correlations between CpGs within promoters or enhancers, and gene expression (Additional file 1: Figure S8). 


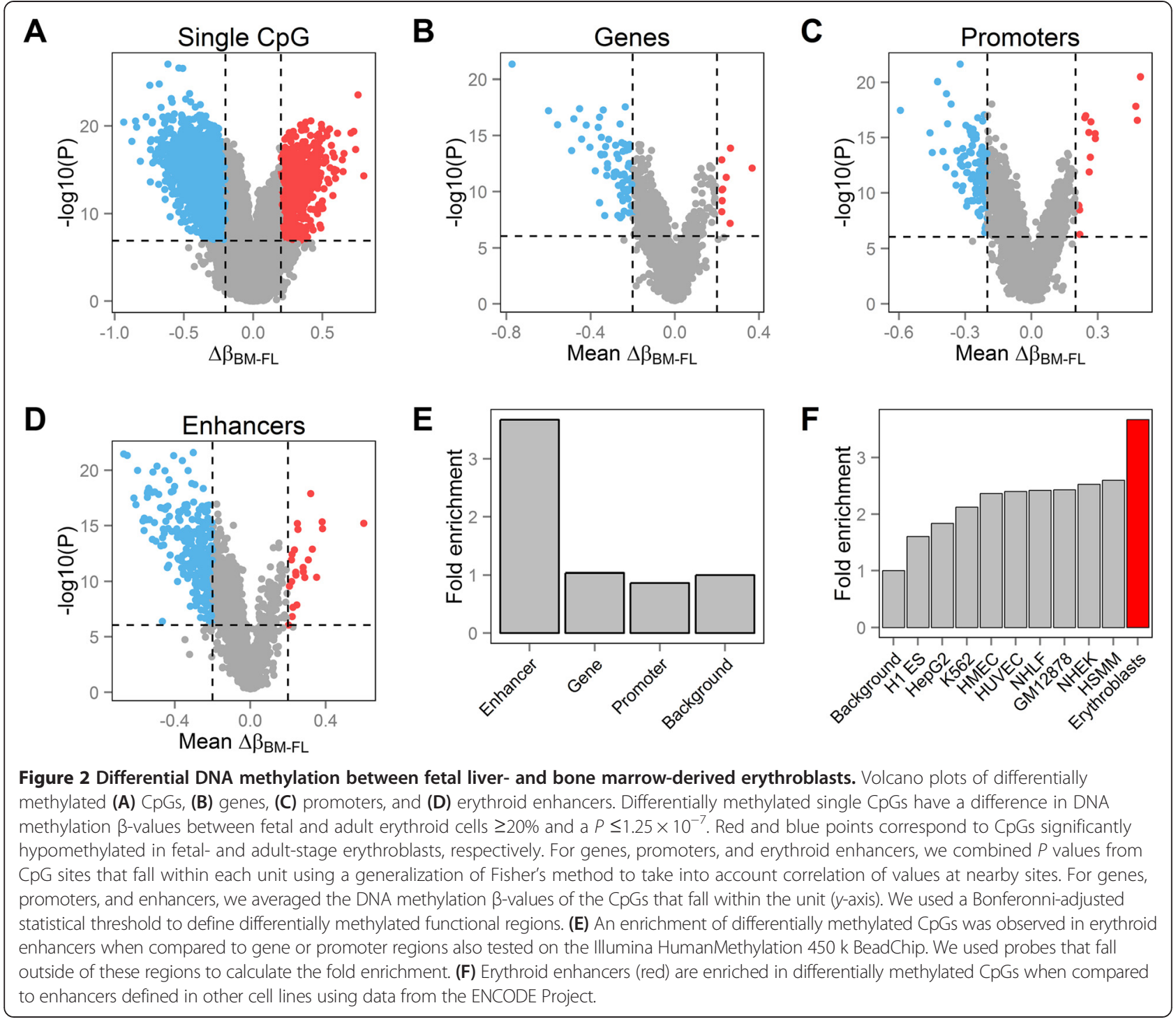

\section{Erythroid enhancers are enriched for differentially methylated CpGs}

To link changes in DNA methylation to biologically relevant functional units, we combined results from single CpGs that fall within each of 25,891 transcripts defined using RefSeq, 25,103 promoters (1.5-kb upstream of RefSeq transcripts) and 4,604 erythroid enhancers [20]. These enhancers, defined using DNase I hypersensitive sites and histone marks, include a set of enhancers that is common to both fetal and adult erythroblasts, as well as developmental lineage-specific enhancers [20]. After Bonferonni correction (significance threshold set at $P<9 \times 10^{-7}$ ), we found 77 genes, 116 promoters, and 303 erythroid enhancers that are differentially methylated (Figure 2B-D and Additional file 3: Table S3).

These analyses highlighted multiple regions of interest (Additional file 3: Table S3). For instance, C22orf26 was one of the most differentially methylated genes (mean
$\left.\Delta \beta_{\text {adult-fetal }}:-0.60 ; P=6.2 \times 10^{-18}\right)$. Although nothing is known about this gene, it is located just upstream of the microRNA let7-b host gene (MIRLET7BHG; Figure 3E). The microRNA let-7b belongs to the let-7 family, is highly expressed in adult as compared to fetal erythroblasts, and correlates with BCL11A expression and reduced $\mathrm{HbF}$ levels [39]. It is possible that DNA methylation at CpGs within or near C22orf26 regulates the expression of MIRLET7BHG. Notably, a nearby enhancer active in adult-stage erythroblasts contain differentially methylated CpGs, which correlates with MIRLET7BHG expression (Figure 3F). Although the CpGs also correlates with C22orf26 expression levels (Figure 3G), this gene is an order of magnitude less expressed, and other CpGs in its core promoter might also control its expression. Finally, we carried out a pathway-based analysis to identify group of functionally related genes that are differentially methylated. We found multiple pathways 


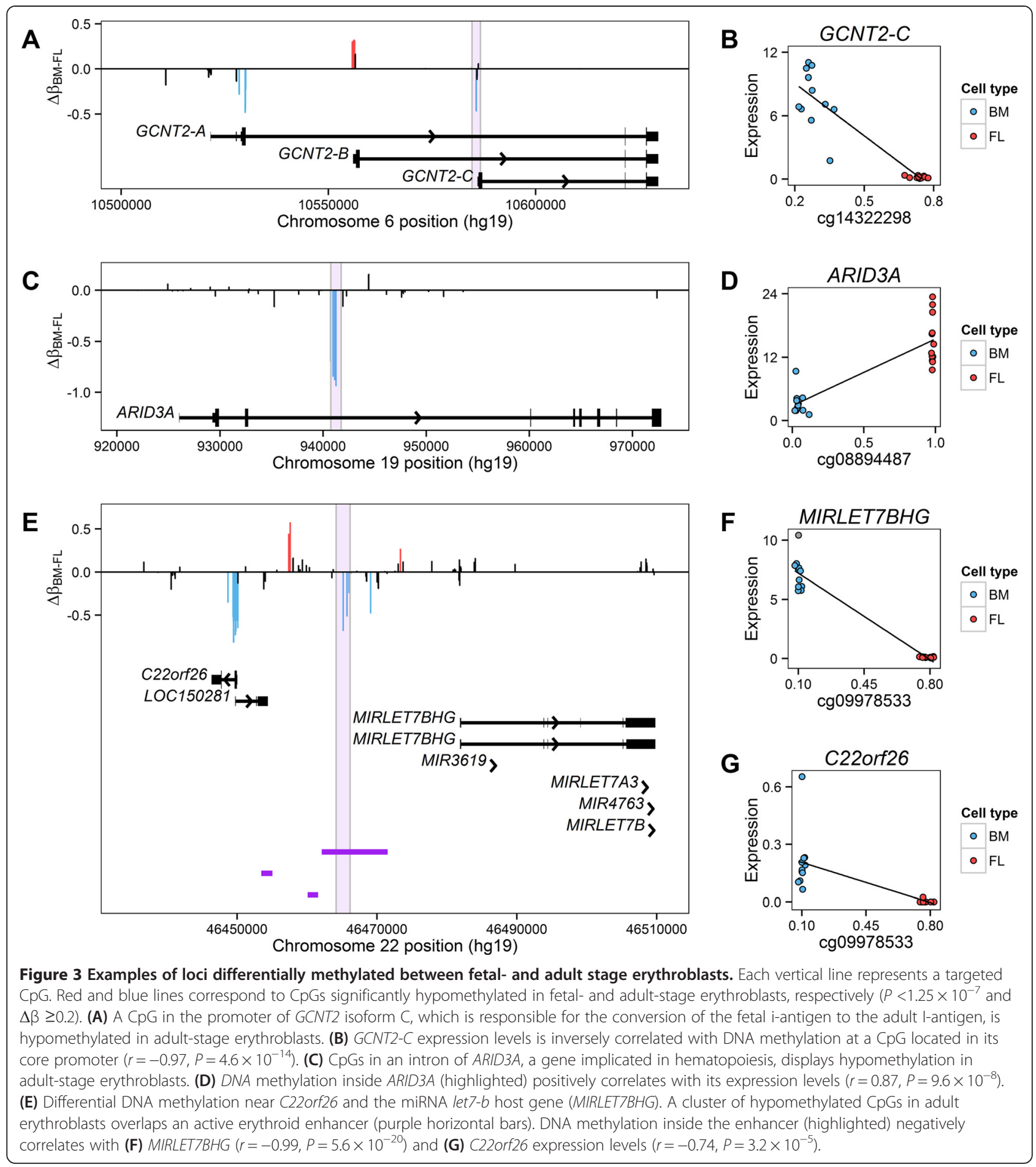

enriched for signaling activity, wound healing, oxygen, cytokine production, circulation, and cation transport (Additional file 4: Table S4 and Additional file 1: Table S5). Many of these pathways include genes not associated with erythropoiesis previously, and we need to validate if differential DNA methylation translates into biologically important functions.
More generally, we asked whether there was an enrichment of differentially methylated CpGs in genes, promoters, and erythroid enhancers when compared to the rest of the genome tested by the Illumina HumanMethylation $450 \mathrm{k}$ BeadChip. We observed a strong statistically significant enrichment of differentially methylated CpGs in erythroid enhancers (3.67- 
fold, $P=9.5 \times 10^{-164}$ ) (Figure $2 \mathrm{E}$ ). The enrichment was marginally significant in genes $(1.03$-fold, $P=0.04)$ and we noted a significant depletion of differentially methylated CpGs in promoters (0.86-fold, $P=4.3 \times 10^{-10}$ ) (Figure 2E). The results for promoters and genes are challenging to interpret given the ascertainment bias in the design of the methylation array. However, this technical confounding does not affect the result for the erythroid enhancers since the release of the Illumina HumanMethylation $450 \mathrm{k}$ BeadChip pre-dated the publication of the erythroid enhancers [20]. Therefore, differential DNA methylation at erythroid enhancers likely captures transcriptional and developmental differences between fetal and adult erythroblasts.

We then compared enrichment of differentially methylated CpGs in enhancers defined in nine different cell types using data from the ENCODE Project [22]. Although there was enrichment at enhancers from other cell types - perhaps suggesting constitutive regulatory functions - we observed the strongest enrichment for erythroid enhancers defined in differentiated CD34+ cells (significant against enhancers from all other cell types, $P<4.5 \times 10^{-17}$, Figure 2 F) [20]. The enrichment of differentially methylated $\mathrm{CpGs}$ within enhancers defined by ENCODE in erythroleukemic K562 cells was similar to the enrichment observed in other, non-hematopoietic cells (Figure 2F). This result may highlight a limitation in using K562 cells to study the fine regulatory mechanisms that control erythropoiesis.

Having demonstrated an enrichment of differentially methylated CpGs in erythroid enhancers, we were interested in testing if these genomic regions are more likely to contain genetic variants associated with $\mathrm{HbF}$ levels. We analyzed associations between HbF levels measured at baseline and genotypes at 6,994,357 markers in 1,140 adult patients with sickle cell disease [28]. As expected, we observed a strong deviation from the null distribution owing to variants in the BCL11A,HBS1L-MYB, and $\beta$-globin loci (Figure 4). When we considered only the 63,876 SNPs that overlap with erythroid enhancers, we again observed deviation from the null expectation. This departure from the null was even true - although more modest - after excluding enhancers near the known $\mathrm{HbF}$ regulators (Figure 4). This result directly implicates erythroid enhancer in inter-individual HbF levels variation, and suggests that future genetic experiments to find new $\mathrm{HbF}$ regulators should focus on these genomic regions.

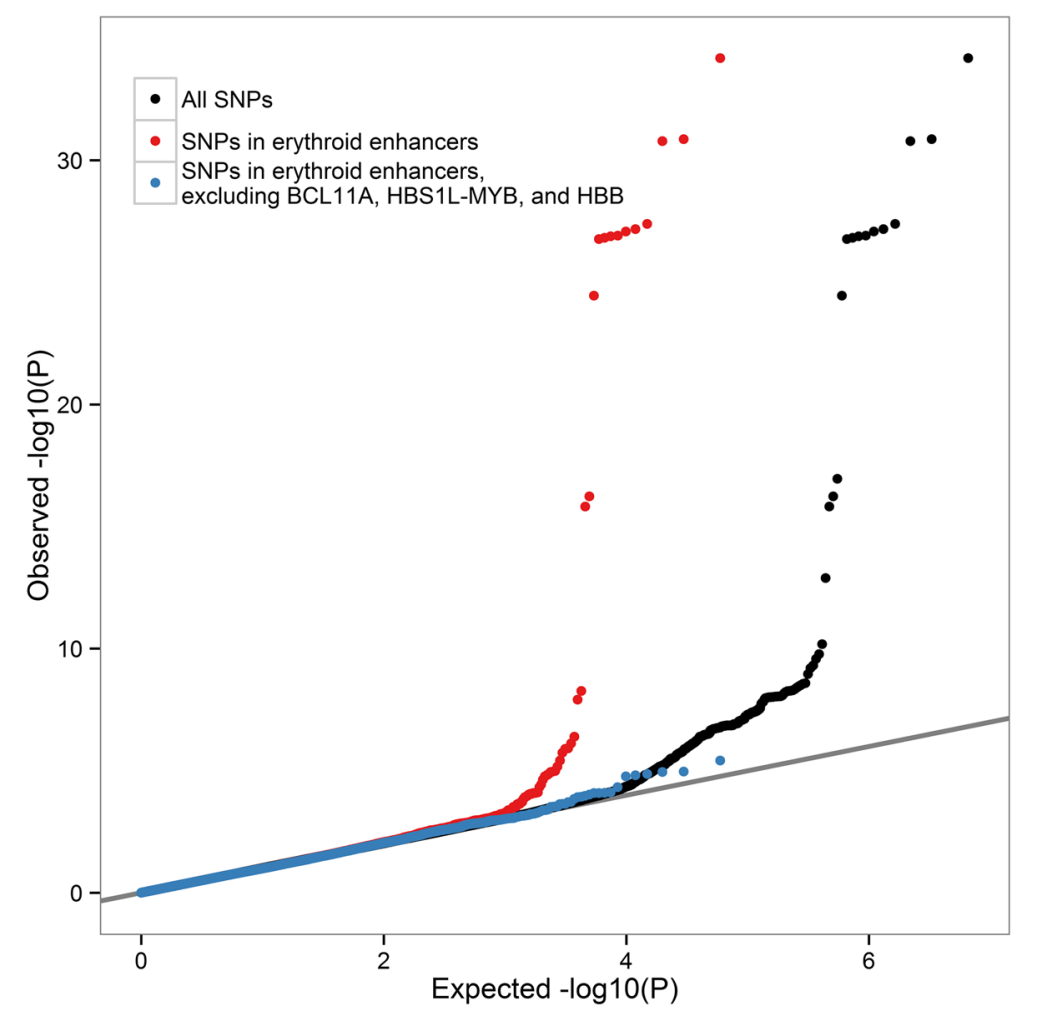

Figure 4 Erythroid enhancers are enriched for SNPs associated with fetal hemoglobin (HbF) levels in patients with sickle cell disease. Quantile-quantile (QQ) plot of association $P$ values with HbF levels in 1,140 sickle cell disease patients. The QQ plot for all 6,994,357 imputed SNPs is shown in black $\left(\lambda_{G C}=1.01\right)$. In red are the 63,876 markers that map to erythroid enhancers $\left(\lambda_{G C}=1.03\right)$ and in blue are the markers that remain after excluding enhancers near BCL11A, HBS1L-MYB and the $\beta$-globin locus $\left(\lambda_{G C}=1.02\right)$. 
Several transcription factor binding motifs are preferentially located near differentially methylated CpGs Data from the ENCODE Project support a passive role for DNA methylation in controlling gene expression: when a transcription factor is not or less expressed, the CpGs surrounding its consensus binding sites tend to be less accessible to DNase I digestion and to be more methylated [40]. Based on this observation, we reasoned that an analysis of transcription factor binding sites within loci that are differentially methylated between fetal and adult erythroblasts might yield new transcription factors important for erythroid development. We performed this analysis on the 5,000 most hypomethylated CpGs in fetal erythroblasts and compared them to the 5,000 most hypomethylated CpGs in adult erythroblasts. We obtained consistent results when using different thresholds. We included 200 base pairs on each side of the CpGs and considered 495 transcription factor binding motifs defined by the HOMER software [25]. We identified enrichment for many transcription factors involved in different cellular processes, including many with known roles during erythropoiesis (Additional file 1:
Tables S6-S7). In Table 1, we list the top transcription factors with binding motifs enriched near hypomethylated CpGs.

In fetal erythroblasts, we observed enrichment for the binding sites of SOX6 and GATA1, two key transcription regulators of hematopoiesis [10,41,42]. GATA binding motifs are also enriched at fetal-specific erythroid enhancers [20]. GATA1 and SOX6 are two transcription factors important in fetal and adult erythroblasts, although our results suggest that they may preferentially bind hypomethylated sites in fetal erythroblasts [43]. The motif recognized by NFY was enriched near CpGs hypomethylated in fetal erythroblasts: NFY binds the HBG2 gene promoters to stimulate chromatin opening [44]. In adult erythroblasts, we detected an enrichment for the motif bound by NF-E2, a transcription factor important for erythroid maturation and $H B B$ gene expression [45], as well as RUNX1, an important regulator of mammalian hematopoiesis that acts upstream of NF-E2 [46]. IRF2 was recently established as a transcriptional regulator of erythropoiesis that controls gene expression through adult erythroid enhancers [20]. Binding sites for

Table 1 Enrichment of transcription factor binding sites (TFBS) near differentially methylated CpGs

\begin{tabular}{|c|c|c|c|c|}
\hline Motif name & Consensus & $P$ value & $q$-value & Fold enrichment \\
\hline \multicolumn{5}{|c|}{ TFBS enriched near CpGs hypomethylated in fetal erythroblasts } \\
\hline SOX2 & BCCATTGTTC & $1.0 \times 10^{-13}$ & 0 & 1.5 \\
\hline TCF3 & ASWTCAAAGG & $1.0 \times 10^{-8}$ & 0 & 1.8 \\
\hline REST-NRSF & GGMGCTGTCCATGGTGCTGA & $1.0 \times 10^{-8}$ & 0 & 5.5 \\
\hline sox6 & CCATTGTTNY & $1.0 \times 10^{-8}$ & 0 & 1.3 \\
\hline GATA1 & AGATGKDGAGATAAG & $1.0 \times 10^{-5}$ & 0.0001 & 2.4 \\
\hline MAZ & GGGGGGGG & $1.0 \times 10^{-5}$ & 0.0002 & 1.1 \\
\hline TCF4 & ASATCAAAGGVA & $1.0 \times 10^{-5}$ & 0.0002 & 1.4 \\
\hline FOXA1 & WAAGTAAACA & $1.0 \times 10^{-5}$ & 0.0002 & 1.3 \\
\hline HNF6 & NTATYGATCH & $1.0 \times 10^{-4}$ & 0.0004 & 1.4 \\
\hline TCFL2 & ACWTCAAAGG & $1.0 \times 10^{-4}$ & 0.0005 & 1.9 \\
\hline \multicolumn{5}{|c|}{ TFBS enriched near CpGs hypomethylated in adult erythroblasts } \\
\hline NF1 & CYTGGCABNSTGCCAR & $1.0 \times 10^{-211}$ & 0 & 3.9 \\
\hline IRF2 & GAAASYGAAASY & $1.0 \times 10^{-78}$ & 0 & 7.3 \\
\hline TLX/NR2E1 & CTGGCAGSCTGCCA & $1.0 \times 10^{-76}$ & 0 & 2.4 \\
\hline NF1-halfsite & YTGCCAAG & $1.0 \times 10^{-56}$ & 0 & 1.4 \\
\hline ISRE & AGTTTCASTTTC & $1.0 \times 10^{-54}$ & 0 & 7.5 \\
\hline $\mathrm{BACH} 1$ & AWWNTGCTGAGTCAT & $1.0 \times 10^{-42}$ & 0 & 6.4 \\
\hline RUNX1 & AAACCACARM & $1.0 \times 10^{-37}$ & 0 & 1.6 \\
\hline RUNX2 & NWAACCACADNN & $1.0 \times 10^{-36}$ & 0 & 1.7 \\
\hline NRF2 & HTGCTGAGTCAT & $1.0 \times 10^{-36}$ & 0 & 5.8 \\
\hline$c-J U N$ & GATGASTCATCN & $1.0 \times 10^{-33}$ & 0 & 2.3 \\
\hline
\end{tabular}

For these analyses, we used the HOMER software and analyzed TFBS located near CpGs ( \pm 200 base pairs) that are hypomethylated in fetal or adult erythroblasts. The top 10 enriched motifs are shown here for each cell type; see Additional file 1: Tables S6-S7 for the complete list of significant TFBS. We calculated $q$-values using the Benjamini-Hochberg method. We calculated the fold enrichment by comparing the number of hypomethylated CpGs near a given TFBS in fetal and adult erythroblasts. The consensus motif follows the IUPAC nomenclature when more than one base is possible. 
NRF2, a transcription factor closely related to NF-E2, are also enriched near hypomethylated CpGs in adult erythroblasts. NRF2 is a transcriptional activator of the antioxidant response and its pharmacological induction in $\mathrm{K} 562$ cells results in increased $\mathrm{HbF}$ production [47]. The most enriched motif in adult erythroblasts belongs to NF1, a family of transcription factors composed of NFIA, NFIB, NFIC, and NFIX. Several differentially methylated CpGs are located near NFIA, NIFC, and NFIX (Additional file 2: Table S2), suggesting that the expression of these transcription factors, as well as their target genes, may be developmentally regulated by DNA methylation during erythropoiesis. Over-expression of NFIA in CD34+ cells leads to increased $H B B$ gene expression [48].

\section{DNA methylation and genetic variation control HBG2 expression}

Treatment with DNA demethylating agents like 5azacytidine induces $\mathrm{HbF}$ production in primates and humans $[49,50]$. The mechanism implies demethylation of the HBG2 promoter, directly or indirectly through an effect on cellular stress, that results in an increased synthesis of $\gamma$-globin chains $[11,12]$. However, it is unknown whether changes in DNA methylation at loci unlinked to the $\beta$-globin cluster on chromosome 11 can also influence the production of $\mathrm{HbF}$ in humans. To explore this hypothesis, we tested if common DNA sequence variants associated with $\mathrm{HbF}$ production in humans are also associated with changes in DNA methylation levels, that is if they are methylation quantitative trait loci (meQTL). Indeed, recent findings suggest that SNPs associated with complex diseases or traits may exert their phenotypic effect by altering DNA methylation profiles [51,52].

We only analyzed associations between $\mathrm{HbF}$-associated SNPs and DNA methylation levels at the BCL11A (chr2: 60,678,302-60,781,633), HBS1L-MYB (chr6:135,281,517135,540,311), and $\beta$-globin (chr11:5,246,696- 5,527,882) loci in adult erythroblasts. We did not include fetal erythroblasts in this analysis because we reasoned that these SNPs affect $\mathrm{HbF}$ production in adult erythroid cells. We complemented the $450 \mathrm{k}$ DNA methylation data with measures of DNA methylation at CpGs in the HBG2 promoter obtained using the targeted assay described above (Additional file 1: Figure S3). We also measured by quantitative PCR the expression of $H B G 2$ and $H B B$ in the same adult cells. In our small sample size $(\mathrm{N}=12)$, we did not identify HbF-associated SNPs that are significantly associated with DNA methylation after accounting for the number of tests performed (Additional file 5: Table S8). Focusing on the $\beta$-globin locus, we noted that increased DNA methylation in the $H B G 2$ promoter was associated with a decreased $H B G 2 / H B B$ gene expression ratio, as expected (Table 2). When we included in the prediction model both DNA methylation levels at the HBG2 promoter and genotypes at rs3759074, both terms were nominally associated in the expected direction with the HBG2/ $H B B$ expression ratio (Table 2). rs3759074 is in linkage disequilibrium with the rs7482144-XmnI variant $\left(r^{2}=\right.$ 0.74) and falls within the BCL11A binding site identified in a functional element important for $\mathrm{HbF}$ silencing [53]. In a mouse model, inactivation of BCL11A and treatment with demethylating 5-aza-2'-deoxycytidine has a synergistic effect on $\mathrm{HbF}$ production [21]. Together with these observations, our results suggest that changes in DNA methylation at the $H B G 2$ promoter act partly independently from genotypes at the $\beta$-globin locus to control $\mathrm{HbF}$ production.

\section{Conclusions}

We generated comprehensive maps of DNA methylation in human erythroblasts differentiated ex vivo from fetal liver or bone marrow CD34+ progenitor cells. At single base pair resolution, we identified 5,937 differentially methylated CpGs that capture many of the transcriptional differences - in terms of transcriptional enhancers and transcription factor binding - between fetal- and adult-stage cells. These analyses also revealed multiple regions that could be of importance for $\mathrm{HbF}$ regulation, as indicated by the enrichment of SNPs strongly associated with $\mathrm{HbF}$ levels within erythroid enhancers. Most of the differentially methylated CpGs are hypermethylated in fetal erythroblasts. We can explore further these

Table 2 Genetic and epigenetic control of HBG2/HBB expression in adult erythroblasts

\begin{tabular}{|c|c|c|c|c|}
\hline \multirow[t]{2}{*}{ Model } & \multicolumn{2}{|c|}{ rs3759074-A } & \multicolumn{2}{|c|}{ CpG (chr11:5,276,172) } \\
\hline & BETA (SE) & $P$ & BETA (SE) & $P$ \\
\hline Model 1: $\log (H B G 2 / H B B) \sim$ rs3759074 & $0.98(0.42)$ & 0.04 & - & - \\
\hline Model 2: $\log (H B G 2 / H B B) \sim C p G$ & - & - & $-6.1(2.0)$ & 0.01 \\
\hline Model 3: $\log (H B G 2 / H B B) \sim \operatorname{rs} 3759074+C p G$ & $5.4(1.6)$ & 0.008 & $-0.81(0.30)$ & 0.02 \\
\hline
\end{tabular}

The A-allele at rs3759074 (chr11:5,257,778) is associated with increased fetal hemoglobin production by genome-wide association studies. In adult erythroblasts, rs3759074-A is associated with increased expression of HBG2/HBB (Model 1). DNA methylation at a CpG located in the HBG2 promoter (chr11:5,276,172) is inversely correlated with HBG2/HBB expression (Model 2). Both genotypes at rs3759074 and DNA methylation at CpG (chr11:5,276,172) are independent predictors of HBG2/ $H B B$ expression levels (Model 3). For this analysis, we used adult erythroblasts from 12 donors. Within this dataset, the rs3759074-A allele frequency is $27 \%$ and the mean DNA methylation value at the tested $\mathrm{CpG}$ is $61 \pm 8 \%$. BETA: arbitrary gene expression units; SE: Standard error. 
DNA methylation differences to understand what distinguishes fetal from adult erythroblasts during human erythropoiesis.

Our study has some limitations. First, the technology that we used to measure DNA methylation does not distinguish between methylation (5-methylcytosine, 5-mC) and hydroxymethylation (5-hydroxymethylcytosine, 5$\mathrm{hmC}$ ). This may be important to functionally explore as 5 - $\mathrm{mC}$ and 5 -hmC have different reported roles in the context of gene regulation [54]. Second, some of the differences observed in DNA methylation levels between fetal and adult erythroblast may be due to slight differences in their growth kinetics. However, we expect this number to be low since our cell morphology and gene expression analyses indicate that these cells are largely undistinguishable. Finally, our analysis of the effect of genetic variation and DNA methylation on globin gene expression is limited by our small sample size. Although our results are consistent with the literature, validation in independent samples is needed to confirm our additive model.

Clinically, one of the most important features of these fetal and adult erythroid cells is their respective production of $\mathrm{HbF}$ or $\mathrm{HbA}$. In patients with sickle cell disease or $\beta$-thalassemia, increasing $\mathrm{HbF}$ production improves disease outcomes. In clinical trials, DNA demethylating agents have shown modest efficacy in increasing $\mathrm{HbF}$ production in patients $[49,50]$. On the other hand, work in a sickle cell mouse model has shown that BCL11A-mediated repression and 5-aza-2'-deoxycytidine treatment synergistically control $\mathrm{HbF}$ production and improve hematological parameters [21]. This is consistent with our observation that a SNP within a BCL11A binding site in a key regulatory element at the $\beta$-globin locus and DNA methylation are independent predictors of $H B G 2$ expression in adult erythroblasts. Together, the mouse and human erythroblast results suggest that a combined strategy to inactivate $B C L 11 A$ and promote $H B G 2$ demethylation may provide the robust induction of $\mathrm{HbF}$ production necessary to treat $\beta$-hemoglobinopathy patients.

\section{Additional files}

Additional file 1: Supplemental data. Supplementary Figures S1 to S8, and supplementary Tables S1, S5, S6, and S7.

Additional file 2: Table S2. Differentially methylated CpGs between ex vivo differentiated fetal and adult erythroblasts.

Additional file 3: Table S3. Genes, promoters, and erythroid enhancers that are differentially methylated when comparing fetal and adult erythroblasts.

Additional file 4: Table S4. Pathways enriched in differentially methylated genes.

Additional file 5: Table S8. Association analysis of

HbF-associated SNPs with DNA methylation at the HBB, BCL 11A, and MYB loci.

\section{Abbreviations}

BM: Erythroblasts derived from bone marrow; FL: Erythroblasts derived from fetal-liver; GO: Gene ontology; HbA: Adult hemoglobin; HbF: Fetal hemoglobin; MALDI-TOF: Matrix-assisted laser desorption/ionization time-of-flight mass spectrometer; meQTL: Methylation quantitative trait locus; miRNA: micro-RNA; qPCR: Quantitative polymerase chain reaction; SNP: Single nucleotide polyphormism.

\section{Competing interests}

The authors declare that they have no competing interests.

\section{Authors' contributions}

SL performed cell culture and molecular biology experiments, carried out bioinformatic and statistical analyses, and wrote the manuscript. MB performed cell culture experiments. KB quantified hemoglobin in primary erythroblasts using capillary electrophoresis. GL conceived of the study, participated in its design and coordination and wrote the manuscript. All authors read and approved the final manuscript.

\section{Acknowledgments}

We thank Ekat Kritikou and Daniel Bauer for critical reading of this manuscript. We also acknowledge investigators from the Roadmap Epigenomics Project [35] for providing access to the RRBS datasets, and thank Dr. Martin H. Steinberg for kindly providing access to the CSSCD GWAS dataset (funded by the NHLBI STAMPEED). SL holds fellowships from the 'Fonds de Recherche du Québec - Santé' and the 'Fondation Pierre Lavoie'. This work was supported by grants from the Canadian Institute of Health Research (\#123382) and the Canada Research Chair program to GL.

\section{Author details}

${ }^{1}$ Montreal Heart Institute, 5000 Bélanger Street, Montréal, Québec H1T 1C8, Canada. ${ }^{2}$ Faculté de Médecine, Université de Montréal, 2900 Boul. Édouard-Montpetit, Montréal, Québec H3T 1J4, Canada. ${ }^{3}$ Hôpital Maisonneuve-Rosemont, 5415 Boul. de I'Assomption, Montréal, Québec H1T 2M4, Canada.

Received: 31 October 2014 Accepted: 9 December 2014

Published online: 20 January 2015

\section{References}

1. Holliday R, Ho T, Paulin R. Gene silencing in mammalian cells. In: Russo VEA, Martienssen RA, Riggs AD, editors. Epigenetic Mechanisms of Gene Regulation. Cold Spring Harbor, NY: Cold Spring Harbor Laboratory Press; 1996. p. 47-60.

2. Bock C. Analysing and interpreting DNA methylation data. Nat Rev Genet. 2012;13:705-19.

3. Heyn H, Esteller M. DNA methylation profiling in the clinic: applications and challenges. Nat Rev Genet. 2012;13:679-92.

4. Trowbridge JJ, Snow JW, Kim J, Orkin SH. DNA methyltransferase 1 is essential for and uniquely regulates hematopoietic stem and progenitor cells. Cell Stem Cell. 2009;5:442-9.

5. Challen GA, Sun D, Jeong M, Luo M, Jelinek J, Berg JS, et al. Dnmt3a is essential for hematopoietic stem cell differentiation. Nat Genet. 2012;44:23-31.

6. Hogart A, Lichtenberg J, Ajay SS, Anderson S, Center NIHIS, Margulies EH, et al. Genome-wide DNA methylation profiles in hematopoietic stem and progenitor cells reveal overrepresentation of ETS transcription factor binding sites. Genome Res. 2012;22:1407-18.

7. Shearstone JR, Pop R, Bock C, Boyle P, Meissner A, Socolovsky M. Global DNA demethylation during mouse erythropoiesis in vivo. Science. 2011;334:799-802.

8. Ziller MJ, Gu H, Muller F, Donaghey J, Tsai LT, Kohlbacher O, et al. Charting a dynamic DNA methylation landscape of the human genome. Nature. 2013;500:477-81.

9. Yu Y, Mo Y, Ebenezer D, Bhattacharyya S, Liu H, Sundaravel S, et al. High resolution methylome analysis reveals widespread functional hypomethylation during adult human erythropoiesis. J Biol Chem. 2013;288:8805-14.

10. Bauer DE, Kamran SC, Orkin SH. Reawakening fetal hemoglobin: prospects for new therapies for the beta-globin disorders. Blood. 2012;120:2945-53. 
11. Groudine M, Eisenman R, Weintraub H. Chromatin structure of endogenous retroviral genes and activation by an inhibitor of DNA methylation. Nature. 1981;292:311-7.

12. Jones PA, Taylor SM. Cellular differentiation, cytidine analogs and DNA methylation. Cell. 1980;20:85-93.

13. Sankaran VG, Menne TF, Xu J, Akie TE, Lettre G, Van Handel B, et al. Human fetal hemoglobin expression is regulated by the developmental stagespecific repressor BCL11A. Science. 2008;322:1839-42.

14. DB Biologics. [http://www.dvbiologics.com/]

15. Lonza. [http://www.lonza.com/]

16. Maksimovic J, Gordon L, Oshlack A. SWAN: Subset-quantile within array normalization for illumina infinium HumanMethylation450 BeadChips. Genome Biol. 2012;13:R44.

17. Fortin JP, Greenwood CMT, Labbe A: ARRmNormalization: Adaptive Robust Regression normalization for Illumina methylation data. In $R$ package 1.0.0 edition; 2013

18. Du P, Zhang X, Huang CC, Jafari N, Kibbe WA, Hou L, et al. Comparison of Beta-value and M-value methods for quantifying methylation levels by microarray analysis. BMC Bioinformatics. 2010;11:587.

19. MPII - RnBeads. [http://rnbeads.mpi-inf.mpg.de/]

20. Xu J, Shao Z, Glass K, Bauer DE, Pinello L, Van Handel B, et al. Combinatorial assembly of developmental stage-specific enhancers controls gene expression programs during human erythropoiesis. Dev Cell. 2012;23:796-811.

21. Xu J, Peng C, Sankaran VG, Shao Z, Esrick EB, Chong BG, et al. Correction of sickle cell disease in adult mice by interference with fetal hemoglobin silencing. Science. 2011;334:993-6.

22. Ernst J, Kheradpour P, Mikkelsen TS, Shoresh N, Ward LD, Epstein CB, et al. Mapping and analysis of chromatin state dynamics in nine human cell types. Nature. 2011:473:43-9.

23. da Huang W, Sherman BT, Lempicki RA. Bioinformatics enrichment tools: paths toward the comprehensive functional analysis of large gene lists. Nucleic Acids Res. 2009;37:1-13.

24. da Huang W, Sherman BT, Lempicki RA. Systematic and integrative analysis of large gene lists using DAVID bioinformatics resources. Nat Protoc. 2009;4:44-57.

25. Heinz S, Benner C, Spann N, Bertolino E, Lin YC, Laslo P, et al. Simple combinations of lineage-determining transcription factors prime cis-regulatory elements required for macrophage and B cell identities. Mol Cell. 2010;38:576-89.

26. Purcell S, Neale B, Todd-Brown K, Thomas L, Ferreira MA, Bender D, et al. PLINK: a tool set for whole-genome association and population-based I inkage analyses. Am J Hum Genet. 2007:81:559-75.

27. Li Y, Willer C, Sanna S, Abecasis G. Genotype imputation. Annu Rev Genomics Hum Genet. 2009:10:387-406.

28. Solovieff N, Milton JN, Hartley SW, Sherva R, Sebastiani P, Dworkis DA, et al. Fetal hemoglobin in sickle cell anemia: genome-wide association studies suggest a regulatory region in the 5 ' olfactory receptor gene cluster. Blood. 2010;115:1815-22.

29. Sankaran VG, Xu J, Ragoczy T, Ippolito GC, Walkley CR, Maika SD, et al. Developmental and species-divergent globin switching are driven by BCL11A. Nature. 2009;460:1093-7.

30. Borg J, Papadopoulos P, Georgitsi M, Gutierrez L, Grech G, Fanis P, et al. Haploinsufficiency for the erythroid transcription factor KLF1 causes hereditary persistence of fetal hemoglobin. Nat Genet. 2010;42:801-5.

31. Zhou D, Liu K, Sun CW, Pawlik KM, Townes TM. KLF1 regulates BCL11A expression and gamma- to beta-globin gene switching. Nat Genet. 2010:42:742-4

32. Mabaera R, Richardson CA, Johnson K, Hsu M, Fiering S, Lowrey CH. Developmental- and differentiation-specific patterns of human gamma- and beta-globin promoter DNA methylation. Blood. 2007;110:1343-52.

33. Shi L, Lin YH, Sierant MC, Zhu F, Cui S, Guan Y, et al. Developmental transcriptome analysis of human erythropoiesis. Hum Mol Genet. 2014;23:4528-42

34. An X, Schulz VP, Li J, Wu K, Liu J, Xue F, et al. Global transcriptome analyses of human and murine terminal erythroid differentiation. Blood. 2014;123:3466-77

35. Roadmap Epigenomics Project. [http://www.roadmapepigenomics.org/]

36. Inaba N, Hiruma T, Togayachi A, Iwasaki H, Wang XH, Furukawa Y, et al. A novel I-branching beta-1,6-N-acetylglucosaminyltransferase involved in human blood group I antigen expression. Blood. 2003;101:2870-6.
37. Webb CF, Bryant J, Popowski M, Allred L, Kim D, Harriss J, et al. The ARID family transcription factor bright is required for both hematopoietic stem cell and B lineage development. Mol Cell Biol. 2011;31:1041-53.

38. Kulis M, Heath S, Bibikova M, Queiros AC, Navarro A, Clot G, et al. Epigenomic analysis detects widespread gene-body DNA hypomethylation in chronic lymphocytic leukemia. Nat Genet. 2012:44:1236-42.

39. Lee YT, de Vasconcellos JF, Yuan J, Byrnes C, Noh SJ, Meier ER, et al. LIN28B-mediated expression of fetal hemoglobin and production of fetal-like erythrocytes from adult human erythroblasts ex vivo. Blood 2013;122:1034-41.

40. Thurman RE, Rynes E, Humbert R, Vierstra J, Maurano MT, Haugen E, et al. The accessible chromatin landscape of the human genome. Nature. 2012:489:75-82.

41. Xu J, Sankaran VG, Ni M, Menne TF, Puram RV, Kim W, et al. Transcriptional silencing of gamma-globin by BCL11A involves long-range interactions and cooperation with SOX6. Genes Dev. 2010;24:783-98.

42. Orkin SH, Zon LI. Hematopoiesis: an evolving paradigm for stem cell biology. Cell. 2008;132:631-44.

43. Palis J. Primitive and definitive erythropoiesis in mammals. Front Physiol. 2014;5:3.

44. Duan Z, Stamatoyannopoulos G, Li Q. Role of NF-Y in in vivo regulation of the gamma-globin gene. Mol Cell Biol. 2001;21:3083-95.

45. Zhou Z, Li X, Deng C, Ney PA, Huang S, Bungert J. USF and NF-E2 cooperate to regulate the recruitment and activity of RNA polymerase $\|$ in the beta-globin gene locus. J Biol Chem. 2010;285:15894-905.

46. Ichikawa M, Goyama S, Asai T, Kawazu M, Nakagawa M, Takeshita M, et al. AML1/Runx1 negatively regulates quiescent hematopoietic stem cells in adult hematopoiesis. J Immunol. 2008;180:4402-8.

47. Macari ER, Lowrey $\mathrm{CH}$. Induction of human fetal hemoglobin via the NRF2 antioxidant response signaling pathway. Blood. 2011;117:5987-97.

48. Starnes LM, Sorrentino A, Pelosi E, Ballarino M, Morsilli O, Biffoni M, et al. $\mathrm{NFI}-\mathrm{A}$ directs the fate of hematopoietic progenitors to the erythroid or granulocytic lineage and controls beta-globin and G-CSF receptor expression. Blood. 2009;114:1753-63.

49. DeSimone J, Heller P, Hall L, Zwiers D. 5-Azacytidine stimulates fetal hemoglobin synthesis in anemic baboons. Proc Natl Acad Sci U S A 1982;79:4428-31.

50. Ley TJ, DeSimone J, Anagnou NP, Keller GH, Humphries RK, Turner PH, et al. 5-azacytidine selectively increases gamma-globin synthesis in a patient with beta + thalassemia. N Engl J Med. 1982;307:1469-75.

51. Gamazon ER, Badner JA, Cheng L, Zhang C, Zhang D, Cox NJ, et al. Enrichment of cis-regulatory gene expression SNPs and methylation quantitative trait loci among bipolar disorder susceptibility variants. Mol Psychiatry. 2013:18:340-6

52. Grundberg E, Meduri E, Sandling JK, Hedman AK, Keildson S, Buil A, et al. Global analysis of DNA methylation variation in adipose tissue from twins reveals links to disease-associated variants in distal regulatory elements. Am J Hum Genet. 2013:93:876-90.

53. Sankaran VG, Xu J, Byron R, Greisman HA, Fisher C, Weatherall DJ, et al. A functional element necessary for fetal hemoglobin silencing. N Engl J Med. 2011;365:807-14

54. Guibert S, Weber M. Functions of DNA methylation and hydroxymethylation in mammalian development. Curr Top Dev Biol. 2013;104:47-83.

\section{Submit your next manuscript to BioMed Central and take full advantage of:}

- Convenient online submission

- Thorough peer review

- No space constraints or color figure charges

- Immediate publication on acceptance

- Inclusion in PubMed, CAS, Scopus and Google Scholar

- Research which is freely available for redistribution 